\title{
Conceptos prácticos para el tratamiento de las deformidades abdominales complejas
}

\section{Pragmatic management fot the treatment of complex abdominal deformities}

Alberto M. L. CALDEIRA*, Kelly CARRIÓN**, John JAULIS**

Resumen

Introducción y Objetivo. Las grandes deformidades abdominales o las secuelas abdominales quirúrgicas desafían la maestría y la técnica del cirujano. Las dificultades que se abordan en estos pacientes están causadas principalmente por déficit de tejido cutáneo en la región abdominal debido a múltiples cicatrices, adherencias, retracciones, lesiones traumáticas e iatrogénicas.

Este trabajo muestra diferentes enfoques para clasificar y gestionar con éxito algunos de estos casos difíciles manteniendo un componente estético.

Material y Método. Revisamos las deformidades abdominales complejas presentando 3 casos seguidos durante toda su evolución, que ayudan a ejemplificar su abordaje práctico y su tratamiento.

Resultados. La cirugía estética abdominal necesita un enfoque global en relación al contorno corporal. La clasificación de la deformidad de pared de las abdominoplastias complejas comprende una categorización anatómica, dividiendo el abdomen en inferior, medio y superior, con o sin exceso de piel, y facilita el tratamiento quirúrgico subsiguiente. Las técnicas quirúrgicas tienen diferentes enfoques para tratar toda la zona toracoabdominal logrando la reconstrucción de la pared abdominal con un mejor resultado del contorno corporal del paciente. Estos procedimientos incluyen técnicas de expansión tisular, reposicionamiento umbilical, abdominoplastias secundarias, abdominoplastias medianas y abdominoplastias reversas.

Conclusiones. Nuestro planteamiento permite tratar abdómenes complejos a través de un manejo diferente, modificando las estrategias de abordaje y conservando los conceptos básicos para alcanzar el resultado armónico y estético deseado. Proponemos que los cirujanos deben separarse de los enfoques quirúrgicos establecidos, para incorporar otras alternativas.

\begin{tabular}{|lrl} 
Palabras clave & $\begin{array}{l}\text { Abdominoplastia, Abdominoplastia reversa, } \\
\text { Abdominoplastia inversa, Liposucción, Lipoinjerto, } \\
\text { Colgajos abdominales, Quemadura abdominal, Expansores. }\end{array}$ \\
$\begin{array}{l}\text { Nivel de evidencia científica } \\
\text { Recibido [esta versión] }\end{array}$ & 4c Terapéutico \\
Aceptado & 8 agosto/2018 \\
10 septiembre/2018
\end{tabular}

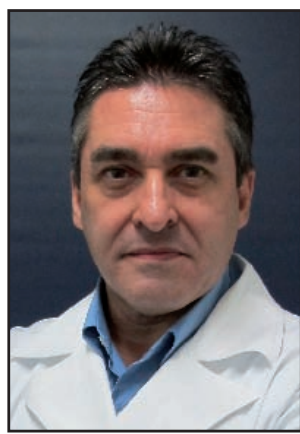

Caldeira, A.M.L.

Abstract

Background and Objective. Big abdominal deformities or abdominal surgical sequeals defy surgeon's expertise and technique. The difficulties approaching these patients are caused principally by poor skin tissue in the abdominal region, where multiple scars, adherences, retractions, traumatic and iatrogenic injuries can be found.

This paper shows different approaches to successfully classify and manage some of these difficult cases maintaining an aesthetic component to it.

Methods. We review the complex abdominal deformities presenting 3 cases that were followed throughout their evolution, which help to exemplify the pragmatic management for the treatment of complex abdominal deformities.

Results. Abdominal aesthetic surgery needs a global approach relative to the body contour. The classification of the wall deformity of complex abdominoplasties comprises an anatomical categorization, dividing the abdomen into lower, middle and upper, with or without excess skin, and provides subsequent surgical treatment. Surgical techniques have different approaches to treat the entire thoracoabdominal area by achieving reconstruction of the abdominal wall with a better result of the patient's body contour. These procedures include tissue expansion techniques, umbilical repositioning, secondary abdominoplasty, medial abdominoplasty, and reverse abdominoplasty.

Conclusions. Our proposal can treat complex abdomens through a different management approach changing strategies and preserving the basic concepts to achieve a harmonious and desired aesthetic result. Surgeons must be separated from the established surgical approaches incorporating other alternatives.

\begin{tabular}{|lr}
\hline $\begin{array}{l}\text { Key words } \\
\text { Abdominoplasty, Reverse abdominoplasty, } \\
\text { Inverse abdominoplasty, Liposuction, Lipofilling, } \\
\text { Abdominal burn, Tissue expanders. }\end{array}$ \\
$\begin{array}{lr}\text { Level of evidence } \\
\text { Received [this version] }\end{array}$ & $4 \mathrm{c}$ Therapeutic \\
Accepted & 8 august/2018 \\
\end{tabular}

Conflicto de intereses: los autores declaran no tener ningún interés financiero relacionado con el contenido de este artículo 


\section{Introducción}

La región abdominal representa una de las partes más importantes para la armonía de la estética corporal. Los tejidos de la pared abdominal resultan afectados por diversas condiciones que pueden conducir a deformidades variables. El abordaje quirúrgico de estas deformidades de la pared abdominal representa uno de los desafíos más importantes en el escenario de la Cirugía Plástica. ${ }^{(1)}$

La técnica quirúrgica de elección dependerá del conocimiento, de la cultura médico-quirúrgica y de la destreza del cirujano. Por eso es importante elaborar un plan de reconstrucción que englobe la reparación y reconstitución de los tejidos afectados, el análisis real del defecto y el uso más eficiente posible de los tejidos adyacentes, así como la sustitución del área comprometida por tejido semejante al original. ${ }^{(2)} \mathrm{Y}$ todo ello priorizando el respeto por los límites anatomofisiológicos y proporcionando un volumen tisular adecuado para la remodelación gradual del área afectada. Por tanto, es necesario establecer múltiples secuencias o tiempos operatorios que garanticen una remodelación progresiva del segmento afectado, tanto de forma estética como funcional. ${ }^{(3)}$

La importancia de orientar al paciente sobre la necesidad de llevar a cabo múltiples cirugías para lograr un resultado definitivo y satisfactorio, junto con la adecuada preparación psicológica, es un recurso que el cirujano debe utilizar en el preoperatorio, estableciendo así una fructífera relación médico-paciente que asegure la confianza de este en el procedimiento o procedimientos que se le van a practicar. ${ }^{(4)}$

En este trabajo presentamos la preparación y clasificación de 3 casos clínicos con deformidades inusuales de la pared abdominal y su efecto en la totalidad del contorno corporal, así como las estrategias empleadas para su abordaje buscando resultados estéticos y armónicos.

\section{Material y Método}

Revisamos las deformidades abdominales complejas presentando 3 casos clínicos seguidos durante toda su evolución, los cuales ayudan a ejemplificar el manejo práctico y secuenciado de estos casos inusuales. Todos los pacientes dieron su consentimiento informado antes de su inclusión en el estudio.

Se trata de casos de conducta compleja, difíciles de incluir en ninguna clasificación de las tradicionalmente conocidas. ${ }^{(5-7)}$ Es por ello que, para este trabajo, utilizamos un esquema de clasificación en el que estratificamos las deformidades en 3 categorías de acuerdo con la localización del defecto, para que así el tratamiento pueda estandarizarse tal y como describimos en la Tabla I.

Esta clasificación trata de deformidades abdominales complejas que por lo general afectan a la piel, al tejido adiposo o al sistema músculo-aponeurótico, cuya extensión puede exceder un cuadrante abdominal. Algunas etiologías comunes a estas asimetrías incluyen las cirugías previas o traumas en la región abdominal.

Subclasificamos cada tipo de deformidad en función del exceso de piel que se presente, lo que nos permite definir una estrategia quirúrgica particular para cada subgrupo. Al abordar el subgrupo sin exceso de piel dividimos el tratamiento en un procedimiento de 2 pasos, centrándonos primero en la expansión de la piel y realizando luego la corrección quirúrgica definitiva. Para los casos en los que hay un volumen de piel adecuado a lo necesario para la reconstrucción debido a la flaccidez de otras áreas, el tratamiento quirúrgico se puede lograr en un solo paso.

Presentamos detalladamente estos 3 casos que ejemplifican los tipos de deformidades complejas de la pared abdominal, su clasificación y cómo fueron tratados quirúrgicamente. ${ }^{(8)}$

Tabla I. Clasificación y tratamiento de las deformidades complejas de la pared abdominal

\begin{tabular}{|c|c|c|c|}
\hline Localización & Tipo & Hallazgos clínicos & Tratamiento \\
\hline \multirow{2}{*}{ Abdomen inferior } & \multirow{2}{*}{ I } & $\begin{array}{l}\text { I a. Deformidad compleja sin exceso } \\
\text { de piel }\end{array}$ & $\begin{array}{l}\text { Expansión de piel + Abdominoplastia secundaria } \\
\text { + Reconstrucción umbilical }\end{array}$ \\
\hline & & $\begin{array}{l}\text { I b. Deformidad compleja con exceso } \\
\text { de piel }\end{array}$ & $\begin{array}{l}\text { Abdominoplastia secundaria + Reconstrucción } \\
\text { umbilical }\end{array}$ \\
\hline \multirow{2}{*}{ Abdomen medio } & \multirow{2}{*}{ II } & $\begin{array}{l}\text { II a.Deformidad compleja sin exceso } \\
\text { de piel }\end{array}$ & $\begin{array}{l}\text { Expansión de piel y Lipoabdominoplastia medial } \\
\text { / Lipoabdominoplastia reversa medial } \\
\text { + Reposicionamiento umbilical }\end{array}$ \\
\hline & & $\begin{array}{l}\text { II b. Deformidad compleja con exceso } \\
\text { de piel }\end{array}$ & $\begin{array}{l}\text { Lipoabdominoplastia medial / } \\
\text { Lipoabdominoplastia medial reversa } \\
\text { + Reposicionamiento umbilical }\end{array}$ \\
\hline \multirow{2}{*}{ Abdomen superior } & \multirow{2}{*}{ III } & $\begin{array}{l}\text { III a. Deformidad compleja sin exceso } \\
\text { de piel }\end{array}$ & $\begin{array}{l}\text { Expansión de piel }+ \\
\text { Abdominoplastia reversa }\end{array}$ \\
\hline & & $\begin{array}{l}\text { III b. Deformidad compleja con exceso } \\
\text { de piel }\end{array}$ & Abdominoplastia reversa \\
\hline
\end{tabular}




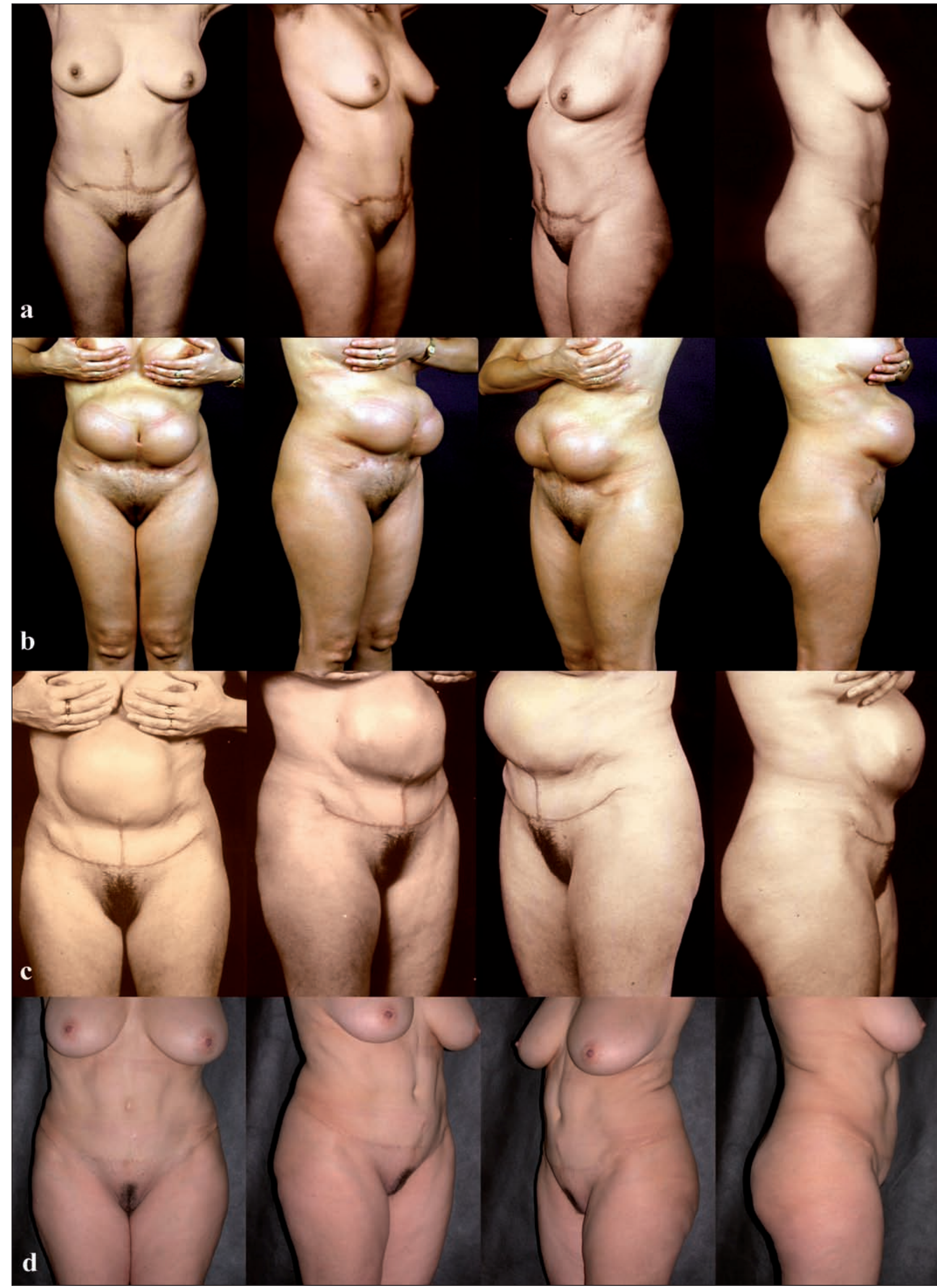

Fig. 1. Tipo I. A. Preoperatorio de la primera intervención quirúrgica. B. Postoperatorio a los 4 meses. C. Postoperatorio a los 3 meses de la segunda intervención quirúrgica. D. Postoperatorio a los 15 años de la tercera intervención quirúrgica. 


\section{RESULTADOS}

Los casos que presentamos a continuación sugieren una clasificación en 3 tipos de deformidad abdominal compleja de acuerdo a sus características y localización.

\section{Tipo I}

Este primer tipo es característico en pacientes con deformidades en la porción infraumbilical del abdomen y deficiencia tisular de la porción superior (Fig. 1 A-D). Para ejemplificar esta categoría presentamos el caso de una mujer de 36 años de edad, con historia de abdominoplastia y onfaloplastia previas con resultado de cicatrices mediales inestéticas infraumbilicales y transversales suprapúbicas, hipertróficas, mal localizadas, con destrucción umbilical, reducción de la altura abdominal y aumento inestético del área pubiana (Fig. 1A).

Como resultado de lo mencionado y del examen físico, planificamos la corrección en 2 o 3 tiempos quirúrgicos diferidos basándonos en la utilización de expansiones cutáneas consecutivas.

En el primer tiempo colocamos 3 expansores tisulares: uno central oval mediano supraumbilical de $800 \mathrm{cc}$ en la región epigástrica, y 2 rectangulares de $600 \mathrm{cc}$ cada uno, localizados respectivamente en las fosas iliacas derecha e izquierda, que expandimos progresivamente durante un periodo de 4 meses, observando una hipoexpansión considerable del implante supraumbilical, por compresión de la válvula remota (Fig. 1B).
A los 5 meses realizamos una abdominoplastia atípica con vaciamiento y retirada de los expansores, plicatura de los rectos abdominales y tracción del colgajo abdominal, seguida de la recolocación de otros 2 expansores vacíos, uno oval de 800 cc en posición medial epigástrica, y otro rectangular de 600 cc en posición supraumbilical, con la intención de que sirvieran para reexpandir el área central mediana en los siguientes meses (Fig. 1C).

En este procedimiento practicamos capsulotomías liberadoras en la cápsula del colgajo dermograso, con la subsecuente tracción y resección de las cicatrices inestéticas y la creación de una cicatriz en ancla convexa localizada debajo de la sínfisis púbica.

Tres meses después de esta segunda intervención, la paciente fue sometida a una nueva abdominoplastia en la cual retiramos los 2 implantes expandidos y obtuvimos una expansión tisular complementaria de aproximadamente $8 \mathrm{~cm}$, que permitió una gran reducción de la cicatriz vertical suprapúbica; realizamos la neo-onfaloplastia utilizando la técnica de Talita Franco ${ }^{(8)}$ (Fig. 1D).

\section{Tipo II}

En este tipo los pacientes presentan deformidades en la porción medial del abdomen y deficiencia tisular (Fig. 2 A y B).

Presentamos como modelo el caso de una paciente de 17 años de edad, con altura de $1.64 \mathrm{~cm}$, peso de $52 \mathrm{~kg}$, e índice de masa corporal (IMC) de 19.3. Presentaba se-

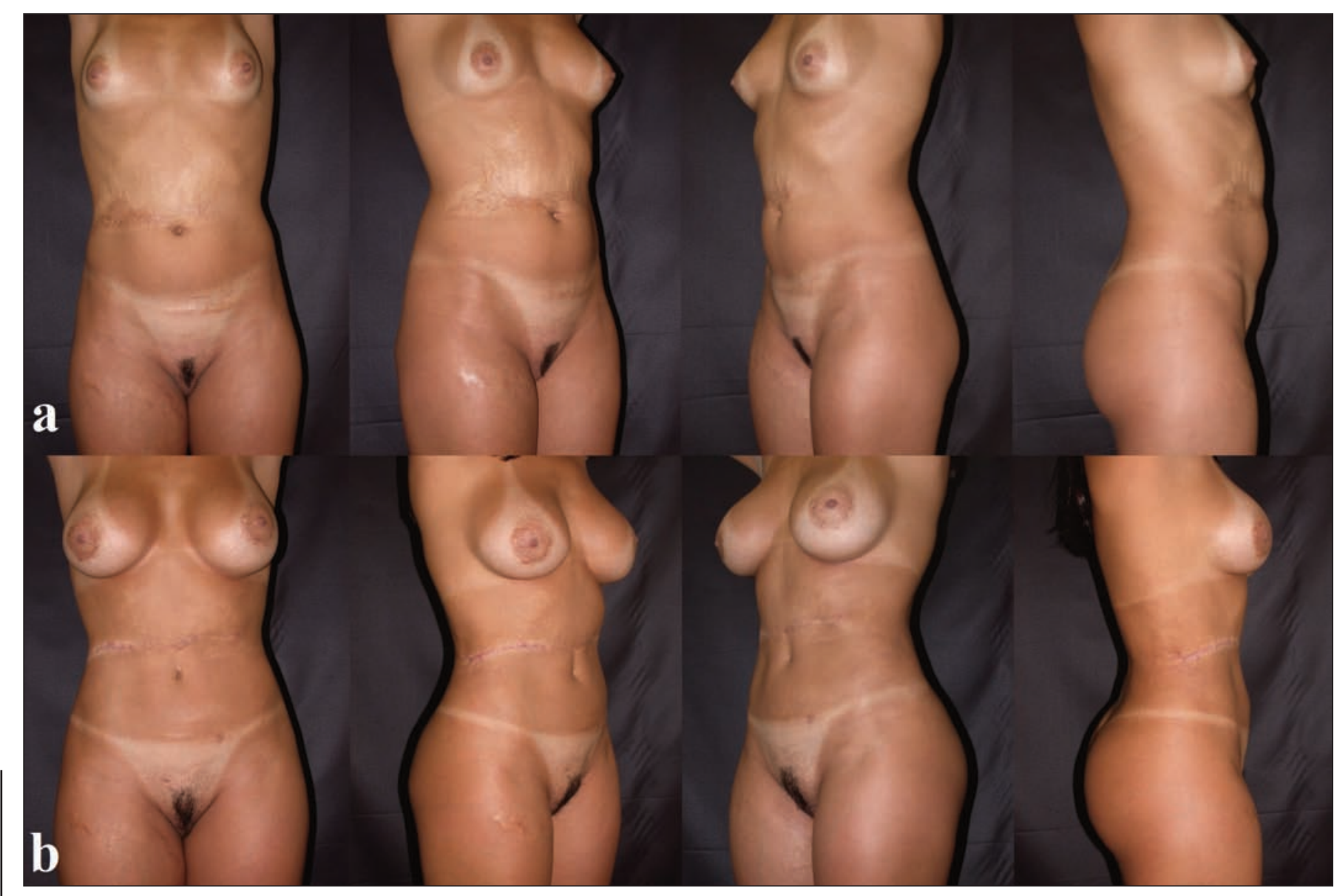


cuela de quemadura en rostro, abdomen y tercio superior de los muslos, refiriendo que a los 4 años de edad había sufrido quemaduras con alcohol incandescente en el $30 \%$ de superficie corporal, con el resultado de cicatrices hipertróficas y queloides en el tercio medial anterior del abdomen (Fig. 2A).

En esta primera evaluación, y debido a que la paciente era adolescente, con poco o ningún tejido adiposo y ausencia de exceso cutáneo, planificamos 3 posibilidades de tratamiento quirúrgico:

- Aumento de peso corporal hasta alcanzar un volu- men adiposo suficiente para expandir la piel y después realizar lipoaspiración, así como generar exceso tisular que permitiera la tracción cutánea adecuada para cubrir el defecto parcial o totalmente.

- Colocación de expansores cutáneos de 1000 a 1500 cc en la región infraumbilical, a fin de lograr expansión progresiva de la piel seguida de una midiabdominoplastia yuxtacicatricial con tracción de la piel expandida para favorecer la resección parcial o total de la cicatriz hipertrófica inestética.
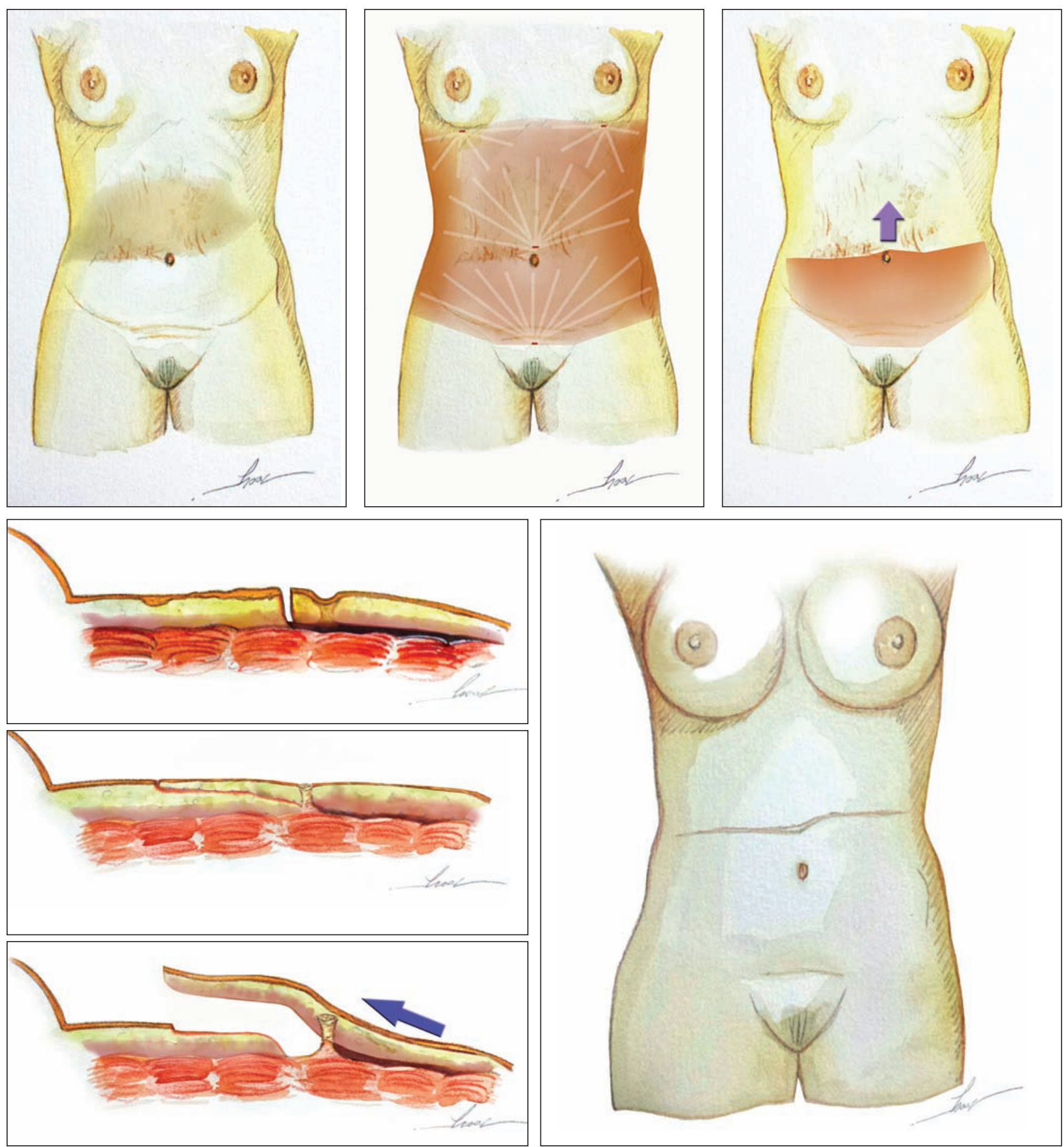

Fig. 3. A. Área con deformidad. B. Liposucción abdominal anterior. C. Disección y creación del colgajo inferior. D-F Avance del colgajo y anclaje de manera superpuesta. G. Resultado final. 
- Conducta expectante hasta después del primer embarazo, momento en el que podríamos utilizar el excedente cutáneo abdominal realizando una miniabdominoplastia reversa con o sin lipoaspiración, con la cual lograríamos la tracción superior del colgajo y la resección de la cicatriz hipertrófica del abdomen supraumbilical.

Tres años después, la paciente retornó a consulta presentando un aumento de peso corporal de apenas $4 \mathrm{~kg}$, con IMC 20.8. Mostraba un razonable aumento de volumen de la grasa subcutánea y un aumento discreto de la elasticidad cutánea abdominal. Teniendo en cuenta la incomodidad de la paciente con su deformidad abdominal, nos decidimos por la primera opción: lipoaspiración de la región dorsolumbar, dorsotorácica, flancos anteriores y posteriores, y del abdomen anterior infraumbilical, seguida por una miniabdominoplastia medial con incisión transversa supraumbilical, yuxtacicatricial, preservando las perforantes vasculares periumbilicales durante la disección, y realizando un avance superior del colgajo lipoaspirado infraumbilical sobre la cicatriz para delimitar el área de resección cicatricial. ${ }^{(9-11)}$ Para sustentar mejor la cicatriz final programamos la sutura de la fascia de Scarpa del colgajo lipoaspirado sobre la fascia de Scarpa del área cicatricial desepitelizada; esta superposición de las fascias superficiales permitiría una mejor calidad de la cicatriz final (Fig. 3).

En este procedimiento, realizamos la lipoaspiración corporal con la técnica super humeda (superwet technique), con infiltración de $2180 \mathrm{cc}$ de solución de Klein, retirada de un volumen total de $3440 \mathrm{cc}$ de grasa que decantamos para obtener $2740 \mathrm{cc}$ de grasa pura que procedimos a injertar en el plano subcutáneo de la región glútea: $300 \mathrm{cc}$ en lado derecho y $280 \mathrm{cc}$ en el izquierdo, empleando para ello cánulas finas, de $2 \mathrm{~mm}$ diámetro, con perforación única.

La lipoaspiración del segmento infraumbilical nos permitió la resección de una pieza de $36 \mathrm{~cm}$ de ancho por $7 \mathrm{~cm}$ de alto que en su mayor parte era tejido hipertrófico. Extirpamos la piel quemada mediante una incisión transversa supraumbilical de Losange gracias a la cual logramos socavar la región infraumbilical, creando una solapa inferior que luego desplazamos superiormente y anclamos a la fascia de Scarpa de la región supra-umbilical, de manera superpuesta. Para completar la lipoabdominoplastia medial sobre la piel subcutánea superior y fijar la fascia de Scarpa del área decorticada, traspusimos la fascia superficial peri-umbilical. Además practicamos una onfaloplastia con remodelación triangular umbilical ${ }^{(9-11)}$ asociada a una lipoescultura corporal en la región sacra, dorsolumbar, flanco posterior y abdomen (Fig. 2B).

\section{Tipo III}

Aquí, las deformidades se presentan en el abdomen superior (Fig. 5 A y B).

Exponemos como ejemplo el caso de una paciente de
35 años de edad con secuelas de mamoplastia previa realizada a los 19 años con resultados insatisfactorios caracterizados por una significativa deflación del contenido mamario bilateral y secuelas de lipoaspiraciones consecutivas realizadas durante los 2 años siguientes, que produjeron un área de $13 \mathrm{~cm}$ de diámetro con adherencias profundas, difusas y transversales en la región supraumbilical, causantes de una distorsión importante, con tracción superior de la cicatriz umbilical que quedaba 17-18 $\mathrm{cm}$ por encima del inicio de la implantación del vello púbico. Como parte del fracaso de los tratamientos realizados, 5 años después la paciente se realizó otra intervención quirúrgica consistente en abdominoplastia reversa con bipartición del tejido graso del colgajo epigástrico y transposición de la grasa bipartida para rellenar el área de adherencia mencionada. Esto creó una nueva deformidad y empeoró las ya existentes, presentando almacenamiento voluminoso de seroma y esteatonecrosis en la región inframamaria (Fig. 5A).

Propusimos una reconstrucción conjunta de la mama y del abdomen en un único tiempo quirúrgico, considerando que el procedimiento de abdominoplastia reversa realizado previamente había creado las condiciones ideales de autonomización del tercio superior del abdomen.

Para ello, procedimos a llevarla a cabo confeccionando un colgajo abdominal, lateral, reverso, de $15 \mathrm{~cm}$ de longitud y $8 \mathrm{~cm}$ de anchura, decorticado en toda su extensión, que introdujimos bajo el músculo pectoral mayor, Tipo II Caldeira, ${ }^{(12-15)}$ para la remodelación mamaria, además de posibilitar la tracción del colgajo supraumbilical con extirpación de un extenso seroma supraumbilical, resección de las cicatrices fibrosas acentuadas y de las áreas de retracción difusas de la zona supraumbilical.

Llevamos a cabo también lipoaspiración con técnica super húmeda de las regiones tóracolateral, tóracodorsal, de los flancos anteriores y posteriores, con infiltración previa de 2500 cc de solución de Klein. Retiramos un volumen total de $4650 \mathrm{cc}$, correspondientes a $3850 \mathrm{cc}$ de grasa pura y decantada, para definir mejor el contorno corporal y la cintura (Fig. 5B).

\section{Discusión}

Toda cirugía reconstructiva debería alcanzar un beneficio efectivamente estético. Asimismo, toda cirugía estética implica un tipo de reconstrucción o reestructuración del contorno corporal. ${ }^{(6,16)}$ Los cirujanos plásticos deberían asumir el papel de innovadores naturales y expertos en la identificación de problemas y en el desarrollo de soluciones únicas para resolverlos. Esta actitud es la responsable de la aparición de nuevas vías y avances de los límites de las fronteras del conocimiento en nuestra especialidad. ${ }^{(17)}$

Siguiendo las nuevas tendencias, la cirugía plástica estética abdominal necesita un enfoque global en relación al contorno corporal. No es razonable abordar el abdomen sin evaluar las regiones y estructuras adyacentes. ${ }^{(6,18)}$ 


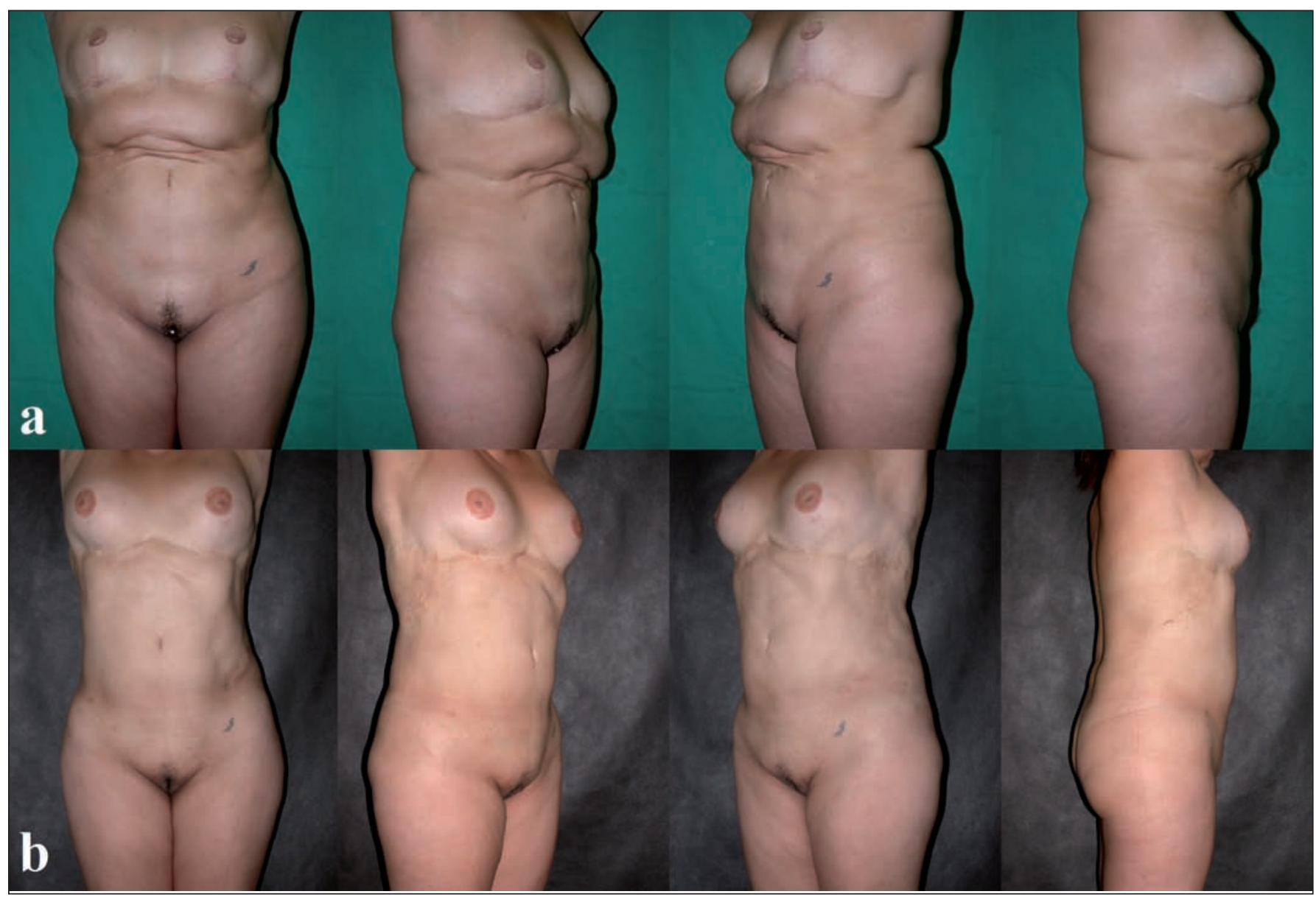

Fig. 5. Tipo III. A. Preoperatorio. B. Postoperatorio a los 8 meses.

Las clasificaciones de las deformidades abdominales publicadas anteriormente las abordan en términos de exceso de piel, depósitos de grasa y laxitud músculo-aponeurótica; ${ }^{(5-7)}$ no tienen en cuenta como sus principales variables la presencia de extensas deformidades cicatriciales, depresiones o retracciones, que sin embargo son el objetivo de este trabajo, por lo cual se presenta la necesidad de categorización de estas deformidades con el fin de estandarizar su foque quirúrgico.

Las obras de Bozola y Psillakis, ${ }^{(5)}$ así como nuestros propios trabajos anteriores ${ }^{(6)}$ y los de Matarazzo ${ }^{(7)}$ han clasificado las deformidades abdominales en términos de gravedad. El tratamiento de estos defectos varía desde la liposucción o miniabdominoplastia sin plicatura para casos más leves, hasta la adición de plicatura infraumbilical para los casos intermedios y la abdominoplastia tradicional para los casos graves asociando lipoaspiración de regiones no socavadas para lograr mejores resultados.

El abdomen es un foco de gran interés en el contorno corporal. Por lo tanto, el enfoque combinado de todas las áreas tóraco-abdominales y el tratamiento adecuado de todas las deformidades presentes, nos permitirá lograr una mejor armonía del contorno corporal. La mayoría de dichas deformidades se pueden encontrar en la región abdominal y están derivadas de factores como la obesidad, grandes pérdidas de peso, múltiples embarazos y secuelas quirúrgicas. ${ }^{(19)}$

Como se ve en el Tipo I de nuestra clasificación a tra- vés del caso que presentamos como ejemplo, la amplitud y las extensión de las cicatrices que comprometían todo el segmento infraumbilical del abdomen, asociadas a notables deficiencias cutáneas y a destrucción umbilical, requirieron del uso de múltiples expansores tisulares simultáneos para poder llevar a cabo distensiones cutáneas consecutivas y lograr la transposición eficaz de la cicatriz final hasta una posición transversal, inferior, cóncava, con sus extremidades bien elevadas, siguiendo el diseño de la ropa de baño. ${ }^{(19-20)}$

Sin duda, un procedimiento simple es lo mejor para un problema simple, pero en una situación más compleja un método simple no puede ser el mejor o es simplemente insuficiente..$^{(6,16)}$

Para seleccionar la ubicación de la cicatriz umbilical que hay que posicionar en el colgajo, utilizamos la distancia ideal entre el ombligo y la implantación del vello púbico, que es de 14 a $16 \mathrm{~cm}$. La técnica utilizada para la neo-onfaloplastia fue una modificación de la técnica de Talita $\mathrm{Franco}^{(8)}$ con retirada efectiva de tejido graso de la región neoumbilical y fijación aponeurótica central circular en la posición previamente determinada.

Está descrito un gran número de procedimientos quirúrgicos que pueden utilizarse para corregir las deformidades abdominales y las de las regiones adyacentes. La evaluación correcta permitirá la elección de los procedimientos adecuados para cada caso en particular. Actual- 
mente, la antigua técnica de abdominoplastia ha sido sometida a modificaciones de adaptación a las nuevas tendencias estéticas. Así, las cicatrices y los desplazamientos tomaron diferentes formas y amplitudes, reduciendo el número de complicaciones y permitiendo mejores resultados estéticos. ${ }^{(21,22)}$ La liposucción, el lipoinjerto y el uso de expansores tisulares complementaron el número de procedimientos utilizados para mejorar el contorno corporal, tratando con mayor precisión cada una de las diferentes deformidades según sus características y requerimientos particulares. ${ }^{(23)}$

En nuestro Tipo II la deficiencia y restricción del avance cutáneo de las cicatrices secundarias por quemadura previa fueron suplantadas por el estímulo de ganancia del peso corporal. Con el aumento de peso logrado por el paciente, nosotros conseguimos la hipertrofia del tejido graso del área circunferencial abdominal generando distensión cutánea, la cual fue suficiente después de la liposucción. El avance del exceso de tejido nos permitió la resección de las áreas de cicatriz hipertrófica mediante una miniabdominoplastia supraumbilical, y la retirada de la cantidad exacta de tejido cicatricial hipertrófico mediante la técnica de onfaloplastia de Avelar. ${ }^{(11)}$ Preservamos y fijamos la fascia superficial (mediante la técnica de superposición de lado a lado) en la reubicación y resección cutáneas, lo cual hizo posible una reestructuración anatómica de la región neo periumbilical de la paciente. ${ }^{(24)}$ Además, empleamos el tejido graso extraído para llevar a cabo gluteoplastia de aumento mediante lipoinjerto, desempeñando un papel fundamental en la redefinición del nuevo contorno corporal. ${ }^{(6,16)}$

En la actualidad, la difusión y popularización de la liposucción como procedimiento importante en la cirugía de contorno corporal, nos ha mostrado en ocasiones resultados muy insatisfactorios motivados por conductas inapropiadas o inadvertidas y procedimientos que provocaron cambios severos o extensos en el tejido graso dérmico abdominal. ${ }^{(13)}$ Las cicatrices quirúrgicas viciosas y de mala calidad, las adherencias, los relieves y la retracción cutánea, son algunos de los múltiples factores que desalientan al cirujano en cuanto al éxito del pronóstico y el tratamiento. ${ }^{(1,11)}$

El Tipo III de nuestra clasificación presentaba deterioro de la pared abdominal debida a 3 liposucciones previas que provocaron áreas de depresión extensa y retracción supraumbilical. La abdominoplastia reversa con bipartición del contenido de grasa suprayacente en bloque movilizado para rellenar el área de retracción, fue un procedimiento absolutamente empírico, erróneo e ineficaz, ya que el bloque de grasa había sufrido por su grosor y extensión, como era de esperar, descomposición total, esteatonecrosis y formación de grandes inclusiones de quistes oleosos. La evolución, sin embargo, promovió una gran extensión de la región abdominal inframamaria y la ganancia de la autonomización de los colgajos abdominales súpero-posteriores. Como consecuencia de estos cambios, pudimos elaborar un plan de reconstitución abdominal y mamaria en el que:

- La abdominoplastia reversa secundaria con reposición de la piel de la parte posterior del abdomen hizo posible la corrección del área de retracción y depresión supraumbilical. ${ }^{(8)}$

- Empleamos la creación de colgajos bilaterales tipo RELAT (colgajo tóracodorsal lateral reverso) ${ }^{(15)}$ utilizando un tejido previamente autónomo para la reconstrucción mamaria fijado internamente en el colgajo bipediculado del músculo pectoral mayor. ${ }^{(12-14)}$

- Realizamos una amplia liposucción del dorso y de los flancos posteriores con el objetivo de reajustar el contorno corporal. ${ }^{(6,16)}$

Consideramos por tanto que las deformidades abdominales deben tratarse junto con las deformidades o cambios de las áreas adyacentes, tales como la región tóracodorsal, los flancos, la mama y la cadera, que son áreas envueltas en el contorno corporal. Al tenerlas en cuenta, podemos realizar un planteamiento lógico de la cirugía del abdomen, armonizado con el resto del contorno corporal como fuente de equilibrio.

Hay casos en los que las deformidades imponen límites y restricciones al abordaje directo basado en los parámetros clásicos descritos para la abdominoplastia. Estos exigen, como hemos mostrado en los casos presentados, que el cirujano modifique los estándares de abordaje e incorpore una conducta novedosa, respetando los principios establecidos para lograr resultados estéticos más refinados y sorprendentes.

Nuestra clasificación para tratar estas deformidades busca estandarizar un tratamiento que dividimos en función de la localización del defecto y de su extensión, pues sabemos que cada segmento se comporta de manera diferente aprovechando las diferentes posibilidades técnicas disponibles. Preconizamos para estos tratamientos la combinación de procedimientos tales como lipoaspiración, uso de expansores, colgajos y otros, pues el uso apropiado y combinado de estas herramientas nos brindará mejores opciones y maximizará nuestros resultados.

\section{Conclusiones}

El problema fundamental de las deformidades de la pared abdominal es la deficiencia de tejidos, caracterizada en general por la falta de piel.

Debemos tomar más en cuenta la liposucción de las áreas de lipodistrofia adyacentes para producir un excedente de piel, con el avance cutáneo, hasta la sustitución del uso de expansores.

Hemos de planificar múltiples tiempos quirúrgicos y diversas técnicas combinadas a la hora de plantear correcciones para restablecer la expresión estética del abdomen y mejorar el contorno abdominal. 


\section{Dirección del autor}

Dr. Alberto M. Lott Caldeira

Instituto de Cirurgia Plástica Avançada Alberto Caldeira

Rua Visconde de Piraja 414 - Ipanema

Rio de Janeiro, Brasil

Correo electrónico: lottcaldeira@gmail.com

\section{Bibliografía}

1. Lockwood T. Fascial anchoring technique in medial thigh lifts. Plast Reconstr Surg. 1988:82(2):299-304.

2. Millard R. Reduction and Esthetic Surgery. En: Guillies H, Millard R. The principles and art of plastic surgery. 1st Ed. Vol II, Little Brown and Company, Boston, 1957. Pp: 390-429.

3. Elbaz JS. Les differents tableaux cliniques. En: Elbaz JS, Flageul G. Cap 3 Liposuccion et Chirurgie Plastique de L'Abdomen. 2da Ed, 1989, Masson, Paris. Pp: 13- 20.

4. Pitanguy I, Caldeira AML, Almeida CC, Alexandrino A. Abdominoplastia - Algumas considerações históricas, filosóficas e psicossociais. Rev Bras Cir. 1982; 72(6):390-402.

5. Bozola AR, Psillakis JM. Abdominoplasty: A new concept and classification for treatment. Plast Reconstr Surg. 1988; 82(6):983-993.

6. Caldeira AML, Niéves A, Oliveira A. Cirurgia do contorno corporal - Reflexões sobre um novo enfoque da cirurgia plástica do abdome. I - Classificações das deformidades abdominais e suas correlações cirúrgicas. Rev Bras Cir 1990; 80(2): 95-104.

7. Matarasso A. Abdominolipoplasty: A system of classification and treatment for combined abdominoplasty and suction-assisted lipectomy. Aesth Plast Surg. 1991; 15:111-121.

8. Franco T. Abdomnioplastias. In: Franco T, Rebello C. Capitulo 9: Cirurgia Estética. Livraria Atheneu, Rio de Janeiro. 1st Ed, 1977; Pp 273-313.

9. Avelar JM. Abdominoplasty - Systematization of a technique without external umbilical scar. Aesth Plast Surg. 1978; 2:141151.

10. Avelar JM. Uma nova técnica de abdominoplastia - Sistema vascular fechado de retalho subdérmico dobrado sobre si mesmo combinado com lipoaspiração. Rev Bras Cir. 1999; 88/89(1/6):3-20.
11. Avelar JM. Abdominoplasty without panniculus undermining and resection: analysis and 3-Years follow up of 97 consecutive cases. Aesth Surg J. 2002; 22(1):16-25.

12. Caldeira AML, Lucas A, Cajano P. Lembi di muscolo gran pettorale nella mastoplastica. Minerva Chir. 1999; 54(10):729738.

13. Caldeira AML, Lucas A, Grigalek G. Mastoplasty: The Triple-Flap Interposition Technique. Aesth Plast Surg 1999; 23:5160.

14. Caldeira AML, Lucas A. Pectoralis Major Muscle Flap: A new support Approach to mammaplasty, personal technique. Aesth Plast Surg. 2000; 24:58-70.

15. Caldeira AML, Barral S, Araújo I, Thomaz D. Aesthetic abdominal and breast reconstruction with reverse lateral thoracodorsal flap. Plast Reconstr Surg 2008; 121(5):346e-347e.

16. Caldeira AML, Niéves A, Oliveira A. Cirurgia do contorno corporal - Reflexões sobre um novo enfoque da cirurgia plástica do abdome II parte. Rev Bras Cir 1990; 80(3):169-186.

17. Caldeira AML, Robles M. Challenges in Abdominoplasty. En: Avelar JM, editor. New Concepts on Abdominoplasty and Further Applications. 1a ed. 2016. Springer, Switzerland. Pp. 481495.

18. Guerrero Santos I, Spaillat L, Morales F, Dicksheet S. Some problems and solutions in abdominoplasty. Aesth Plast Surg. 1980. 4:227-237.

19. Caldeira AML, Lucas A. Flacidez músculo-aponeurótica abdominal intensa y su tratamiento. Cir plást iberolatinoam. 1998; 24(1):9-15.

20. Psillakis JM. Plastic surgery of the abdomen with improvement in the body contour. Physiopathology and treatment of the aponeurotic musculature. Clin Plast Surg 1984; 11(3):465-477.

21. Sinder R. Plastic Surgery of the abdomen - Personal Technique. En: Abstracts of the 6th International Congress of Plastic and Reconstructive Surgery, Masson, Paris. 1975. P. 58.

22. Wilkinson TS, Swartz BE. Individual modifications in body contour surgery: The "limited" abdominoplasty. Plast Reconstr Surg. 1986; 77:779-784.

23. Saldanha OR, Pinto EB, Matos WN Jr, Lucon RL, Magalhães F, Bello EM. Lipoabdominoplasty without undermining. Aesth Surg Journal. 2001; 21: 518-526. DOI: 10.1067/maj.2001.121243

24. Baroudi R, Ferreira CA. Seroma: how to avoid it and how to treat it. Aesth Surg Journal. 1998; 18(6):439-41. 


\title{
Comentario al artículo “Conceptos prácticos para el tratamiento de las deformidades abdominales complejas"
}

\author{
Pedro ARQUERO \\ Especialista en Cirugía Plástica, Estética y Reparadora. Práctica privada, Madrid, España.
}

Tuve el privilegio de acompañar durante algunos años de mi formación al Dr. Alberto Caldeira del que aprendí no sólo las técnicas de nuestro común maestro, Pitanguy, sino también, en un ejercicio de continua disconformidad, a continuar evolucionándolas. Imaginativo, creativo, innovador, siempre se ha atrevido a buscar nuevas soluciones a aquellos problemas que nuestra especialidad va encontrando. Por eso le atraen los retos, porque su verdadera vocación es alcanzar el verdadero oficio de cirujano plástico: reconstruir lo que parece incorregible, las grandes deformidades. Y para eso se necesitan dos cosas: un gran bagaje quirúrgico y una importante dosis de valor.

Entrando en el tema encomendado, diré que Brasil reúne al mas alto número de estudiosos de la abdominoplastia.

No conforme con la abdominoplastia convencional con sus variantes conocidas por todos ${ }^{(1)}$ el trabajo del Dr. Caldeira nos presenta 3 casos en los que destaca la dificultad de resolución y expone la versatilidad necesaria de los 3 tipos de emplazamiento de la incisión transversa: inferior, media y superior, que se corresponden con la localización de las deformidades.

En el primer caso muestra una abdominoplastia secundaria: con cicatrices hipertróficas, defecto de la resección cutánea en el abdomen inferior y carencia de exceso cutáneo en el abdomen superior. Para su tratamiento el Dr. Caldeira ha optado por el camino más difícil: la compleja solución de la expansión tisular.

No cabe duda de que la primera opción sería la realización de una abdominoplastia secundaria con revisión de cicatrices en $\mathrm{T}$ invertida, pero es necesario conocer el alto nivel de exigencia de la mujer brasileña para comprender esta decisión. Solo el rechazo de la cicatriz vertical puede justificar que la paciente acepte varios tiempos quirúrgicos, un largo tiempo de deformidad abdominal, el alto coste y el riesgo de infección, extrusión o rigidez capsular.

Si algo podemos objetar es el emplazamiento inicial de los expansores (muy bajos y laterales) corregido en la segunda ocasión. El resultado es excelente a pesar de no conseguir eliminar por completo la cicatriz vertical suprapúbica,

El segundo caso es una secuela de quemadura que afecta al abdomen medio. En esta ocasión señala los métodos para obtener un excedente cutáneo capaz de cubrir el área cicatricial: engordar, expansores y embarazo. El tiempo y el aumento de peso ayudaron a solucionar el problema.

El tercer caso es aún más complejo. A las secuelas del seroma en el tercio superior del abdomen, ocasionado por las liposucciones previas, se suma una falta de tejido mamario como resultado de una reducción mamaria. Esto lo soluciona con una abdominoplastia invertida y la utilización del excedente cutáneo para el relleno de las mamas mediante un colgajo abdominal lateral decorticado.

En su conjunto, el trabajo propone una clasificación de las deformidades complejas de la pared abdominal; resalta la importancia de la visión conjunta toracoabdominal en la armonía del contorno corporal, incluido el lipoinjerto, especialmente en las capas superficiales del glúteo; también aboga por la utilización de la lipoabdominoplastia al tiempo que advierte de los peligros y limitaciones de la técnica y utiliza el importante concepto de autonomización de los colgajos.

Solo me cabe decir que he echado en falta la mención tanto de los puntos de Baroudi como de la importancia de las alteraciones músculo-apeneuróticas. ${ }^{(2)}$

Finalmente, me gustaría transmitir mi agradecimiento y mi felicitación al Dr. Alberto Magno Lott Caldeira por su generosidad y su afán de superación.

Bibliografía

1. Arquero P. Barros J. Dermolipectomía Abdominal: nuestra conducta. Cirugía Estética Plástica 2009, (9): 42-48.

2. Nahas FX, Ferreira LM. Concepts on correction of the musculoaponeurotic layer in abdominoplasty. Clin Plast Surg. 2010;37(3):527-538. 


\section{Respuesta al comentario del Dr. Aquero}

\section{Alberto M. L. CALDEIRA}

Desde el inicio de mi carrera, tengo establecido que la continua búsqueda de la excelencia debe situarse como principio fundamental de nuestra actividad. Siguiendo mi natural y verdadera vocación por la enseñanza, quiero decirles que el privilegio de trabajar con jóvenes talentosos cirujanos siempre me exigió buscar la persistente actualización de mis conocimientos para encarar de forma responsable y consecuente los desafíos de las grandes deformidades o de aquellas que parecían incorregibles.

Me gustaría esclarecer algunos puntos sobre cada uno de los 3 casos presentados y que fueron comentados por el Dr. Pedro Arquero.

El primer caso es el de una paciente joven, nulípara, con un importante compromiso del abdomen inferior y destrucción de la cicatriz umbilical debido a un equivocado e injustificado procedimiento estético previo. Como cirujanos plásticos presentamos soluciones de acuerdo a las exigencias e insatisfacciones personales de nuestros pacientes. Nosotros respondemos a sus expectativas posibles y deseos factibles. En este caso específico, la expansión tisular fue proyectada para ser hecha en tiempos consecutivos con aprovechamiento y desplazamiento de los mismos expansores iniciales debido a la gran extensión, mediana y vertical, de la cicatriz.

En el segundo caso, la expansión tisular fue promovida por el aumento ponderal con expansión del tejido subcutáneo, que al ser lipoaspirado, permitió el avance del segmento dermograso. Me gustaría resaltar la metodología de anclaje de la fascia superficial del colgajo de avance sobre la fascia superficial del área cicatricial retirada como mecanismo de soporte a la tracción natural del colgajo en dirección a su posición original.
En el tercer caso, la paciente había sido sometida previamente a una abdominoplastia reversa con errónea bipartición del tejido graso. Logramos obtener 2 colgajos dermograsos desepitelizados (RELAT flap - reverse lateral thoracodorsal flap) aprovechando la autonomización promovida por esa previa abdominoplastia reversa. En este caso conseguimos realizar una verdadera reconstrucción del contorno corporal anterior.

La ausencia de citación de los artículos y trabajos de Baroudi y Pollock reside en el hecho de que estos procedimientos figuran como métodos auxiliares de cierre en las diversas técnicas de abdominoplastia.

Las alteraciones músculo-aponeuróticas abdominales no fueron discutidas en este artículo, pues estos pacientes no presentaban alteraciones distinguibles, cuya corrección específica y directa fue mencionada por nosotros en publicaciones precedentes..$^{(1-3)}$

Bibliografía

1. Caldeira, A.M.L., Lucas A. Flacidez Músculo-Aponeurótica Abdominal Intensa. Su Tratamiento. Cir plást iberolatinoam 1998, 24(1): 9-15.

2. Caldeira, A.M.L., Carrion, K., Jaulis, J. Repair of the Severe Muscle Aponeurotic Abdominal Laxity with Allopastic Mesh in Aesthetic Abdominoplasty. Aesth Plast Surg, https://doi;org/10.1007/s00266-018-1101-6.2018.

3. Caldeira, A.M.C, Robles, M.B.M., Challenges in Abdominoplasty. En: J.M. Avelar (Ed.) New Concepts on Abdominoplasty and Further Applications, DOI 10.1007/978-3-319-278513_30.2016. 